\title{
Mothers of children at risk described engaging with home visitors in terms of limiting family vulnerability
}

Jack SM, DiCenso A, Lohfeld L. A theory of maternal engagement with public health nurses and family visitors. J Adv Nurs 2005;49:182-90.

\section{How do mothers of children at risk describe the process of engaging with public health nurses (PHNs) and family visitors (FVs) in a home visiting programme?}

\author{
DESIGN \\ Grounded theory.
}

\section{SETTING}

Southwestern Ontario, Canada.

\section{PARTICIPANTS}

A purposeful sample of 20 English speaking mothers who had received $\geqslant 1 \mathrm{PHN}$ visit and $\geqslant 3 \mathrm{FV}$ visits after they had been identified as high risk because of social or economic factors.

\section{METHODS}

Mothers participated in 1-3 semistructured interviews of $60-90$ minutes, which addressed their experiences and expectations of engagement with PHNs and FVs. 29 interviews were conducted (11 by telephone). Interviewer field notes and client records were also reviewed. Data were analysed using open, selective, and theoretical coding. Checks for data credibility included peer debriefing and member checks by 8 participants.

\section{MAIN FINDINGS}

Mothers described feeling vulnerable because PHNs and FVs had the power to alter family structure. When mothers risked participating in home visits, they engaged in a social process to limit family vulnerability, which involved 3 phases: overcoming fear, building trust, and seeking mutuality. These phases comprised a circular process, each dependent on the establishment and stability of the other 2 phases. The speed at which the phases were successfully negotiated was influenced by the personal characteristics, values, experiences, and actions of the PHN, FV, and mother. (1) Overcoming fear. Mothers were ambivalent about accepting initial PHN visits, fearing that they would be judged to be inadequate. Strategies to overcome this fear included "hiding nothing," "trying to measure up" (eg, cleaning the house before the visit), and "protecting self" (eg, having a spouse present to reduce the likelihood of negative comments from the PHN). Successful use of these strategies allowed mothers to redefine the role of the PHN from authority figure to supportive service provider. Consequences of not overcoming fear included mothers cancelling scheduled visits and dropping out of the home visiting programme without explanation. Most mothers were less fearful of engaging with FVs than PHNs. (2) Building trust. As mothers' trust in home visitors increased, they became more willing to discuss personal, sensitive issues. "Opening up" was easiest if they perceived that the home visitor was reliable, would keep information confidential, and would not react negatively to disclosed information. Mothers who were open to the relationship, motivated to participate, confident in their parenting abilities, and who perceived the PHN as a positive source of support were more likely to immediately trust the PHN or FV. Mothers often tested home visitors, using secondary sources to verify information and assessing how much information visitors would share about other clients. Mothers had more trust in either PHNs or FVs based on individual characteristics, such as

For correspondence: Dr S Jack, School of Nursing, McMaster Üniversity, Hamilton, Ontario, Canada. jacksm@mcmaster.ca

Source of funding: no external funding reliability and empathy, rather than on role. (3) Seeking mutuality. All mothers wanted to be able to openly share their stories with PHNs and FVs and, in turn, wanted home visitors to share personal experiences, especially those related to child rearing. They promoted sharing by offering refreshments and encouraging brief social exchanges. Mothers observed the verbal and non-verbal language of home visitors and felt comfortable engaging with those who smiled, nodded their heads in encouragement, and allowed adequate time for mothers to speak. When mutual exchanges of information occurred, mothers felt less vulnerable in answering personal questions. Mothers tried to build alliances with home visitors to respond to relatives who were overly critical of their parenting abilities. Mothers wanted to collaborate with home visitors to define common goals for home visits. Generally, FVs were reported to be less likely to collaborate and more likely to lecture mothers.

\section{CONCLUSION}

Mothers of high risk children described engaging with public health nurses and family visitors as a circular process, with the goal of limiting family vulnerability by overcoming fear, building trust, and seeking mutuality.

\section{Commentary}

ncreasing evidence from quantitative studies suggests that home visiting programmes for vulnerable families can help to improve outcomes in young children. ${ }^{1}$ Although this research is important for policy development, these studies provide less direction for practitioners about the processes that contribute to, or detract from, the success of the visiting experience. In addition, few studies have examined parents' perspectives of receiving home visiting services.

The study by Jack et al is noteworthy because of the rigorous qualitative design, careful analysis, and detailed reporting of the findings. Participants were mothers of young children receiving services from one Canadian public health unit. However, the findings are relevant to community nurses in similar programmes who deliver and administer services and guide and supervise home visitors. The findings underscore the importance of home visitors engaging with mothers to build and strengthen their relationship during initial and ongoing visits. Although previous authors have advocated the importance of developing relationships, little empirical evidence exists as to what influences relationship work from the perspective of mothers. Understanding mothers' feelings of vulnerability and the processes they use to limit this vulnerability may help home visitors to reduce the high attrition rates and missed visits reported in many home visiting programmes. Developing and evaluating clinical approaches to reduce mothers' feelings of vulnerability have the potential to improve the services home visitors deliver to high risk families. Karen I Chalmers, RN, PhD Senior Scholar Faculty of Nursing, University of Manitoba Winnipeg, Manitoba, Canada Honorary Visiting Professor
School of Nursing, Midwifery and Social Work
University of Manchester University of Manchester Manchester, UK

1 Elkan R, Kendrick D, Hewitt M, et al. The effectiveness of domiciliary health visiting: a systematic review of international studies and a selective review of the British literature. Health Technol Assess 2000;4:i-v, 1-339. 